\title{
Effects of mental resource availability on looming task performance
}

\author{
Austen B. McGuire ${ }^{1}$ • Omri Gillath ${ }^{1}$ - Michael S. Vitevitch ${ }^{1}$
}

Published online: 23 October 2015

(C) The Psychonomic Society, Inc. 2015

\begin{abstract}
Past research has shown that the looming bias - the tendency to judge one's distance to an approaching object as shorter than in actuality - is stronger among people who are physically weak or vulnerable. The current study examined whether the looming bias would also be stronger among people who are mentally weak or vulnerable. We tested that hypothesis by subjecting 46 young adults to cognitive load and examining their perceptions of approaching objects distance. Participants completed two blocks of the looming task, once under high cognitive load (memorizing a seven-digit number) and once under low load (memorizing a two-digit number). Participants exhibited a stronger looming bias under high load than under low load. These findings support the hypothesis that the looming bias will be stronger when people are weak or vulnerable - either physically or mentally - and in need of a larger margin-of-safety.
\end{abstract}

Keywords Looming bias · Cognitive load · Auditory stimuli · Selective attention $\cdot$ Margin-of-safety theory

Changes in one's environment can signify threat or danger (e.g., a predator or enemy). Being able to detect such changes can help an organism prepare for potential threats, and increase its chances of survival (Cole \& Kuhn, 2010). The human perceptual system has evolved to facilitate high sensitivity to changes in the environment (Cacioppo \& Fredberg, 2012). According to the behavioral urgency hypothesis

Austen B. McGuire

austenmcguire@gmail.com

1 Department of Psychology, University of Kansas, 1415 Jayhawk Blvd., Lawrence, KS 66045-7556, USA
(Franconeri \& Simsons, 2003), humans are especially sensitive to stimuli in the environment that require immediate action. That is, humans pay more attention to unfamiliar objects, objects that suddenly move, and approaching objects (see also Abrams \& Christ, 2006).

The looming bias (Neuhoff, Planisek, \& Seifritz, 2009)the tendency to judge one's distance from approaching objects as shorter than in actuality - goes beyond the behavioral urgency hypothesis to suggest that the need for immediate action promotes a biased perception of one's environment. Specifically, to be able to react to looming objects faster, people need to get ready for them; perceiving looming objects as closer than they really are provides an individual with additional time for preparation (Neuhoff, Planisek, \& Seifritz, 2009; Seifritz et al., 2002; von Mühlenen \& Lleras, 2007). The looming bias does not occur due to a lack of ability to evaluate distance or change in loudness of a sound - as evident by individuals' ability to make accurate distance and sound intensity change judgments regarding objects that are moving away from them (Neuhoff, 2001; Neuhoff \& Wess, 1998). Rather it happens due to a unique bias toward potentially dangerous stimuli-looming objects, not stationary or receding ones (Neuhoff, 2001).

The looming bias is often seen as an evolutionary adaptation (Neuhoff, 2001). The fact that the looming bias has also been observed in non-human primates (Ghazanfar, Neuhoff, \& Logothetis, 2002) suggests that it is a primal process with early evolutionary roots (Barkow, Cosmides, \& Tooby, 1995). Further support for this proposition comes from the work of Bach et al. (2008), who found that looming sounds tend to activate parts of the brain thought to be more primitive such as the limbic system (e.g., amygdala, intraparietal sulcus, and superior temporal sulcus). These areas of the limbic system, which is sometimes referred to as the reptilian or mammalian brain (LeDoux, 1994), are associated with threat and danger and are not activated following exposure to receding sounds. 
Similar to other primal functions related to survival (e.g., various motor reflexes, such as quickly pulling your hand away from a hot stove; Waterhouse \& Campbell, 2011), the looming bias should (1) manifest itself mainly when people are in danger, and (2) not be resource dependent, or be effortless/automatic as opposed to effortful (Neuhoff, 2001). In the current paper we therefore examined two questions regarding the mechanism of the looming bias: (1) Is the looming bias likely to be stronger when people are mentally weak or vulnerable, and (2) Is the looming bias an effortful or effortless process?

Neuhoff, Long, and Worthington (2012) provide support for the first proposition, showing that a stronger looming bias is exhibited among people when they are physically vulnerable, or are less capable of coping with incoming danger. Specifically, Neuhoff et al. (2012) found that people who were potentially more vulnerable due to limited physical strength or lower physical fitness, displayed a stronger looming bias compared to individuals who were stronger and more physically fit. This supports the margin-of-safety theory, according to which the more vulnerable someone is, the greater margin of safety he or she needs to prepare for a threat, and hence the bigger the looming bias he or she should have.

While consistent with the margin-of-safety theory (Neuhoff et al., 2012), there is no research to date examining whether people who are mentally weak or more vulnerable also show a stronger looming bias, similar to people who are physically weaker. Initial evidence to support the idea that being mentally vulnerable may result in stronger looming bias comes from research on individuals who are high on general anxiety. People high on anxiety are likely to have only limited mental capabilities to handle a potential threat (Riskind, Kleiman, Seifritz, \& Neuhoff, 2014; Sorg \& Whitney, 1992). Riskind et al. (2014) found that such people exhibited, as expected, a stronger looming bias. However, we do not know whether these highly anxious individuals indeed had limited mental resources, or whether some other factor related to anxiety (e.g., negative mood, or divided attention; Sorg \& Whitney, 1992) led them to exhibit the increased looming bias.

Although being low on mental resources may have similar effects on the looming bias as being low on physical resources (i.e., stronger looming bias), it may also result in the opposite effect, that is, a weaker or even no looming bias. This will happen if the looming bias is effortful-in other words, it necessitates mental resources. If it is mentally effortful, being low on mental resources may result in a weaker looming bias. A second goal of our study therefore was to examine whether the looming bias is resource dependent (effortful) or not (effortless).

A cognitive bias that is costly or heavily dependent on resources is less likely to contribute to one's chances of survival, especially during times of stress or threat when those resources are needed for coping (e.g., fight or flight;
Edmunds, 1974). In contrast, a bias that is effortless or requires fewer resources is advantageous and more likely to increase one's chances of survival (Neuhoff et al., 2012). Indeed, Neuhoff et al. (2012) suggested that the looming bias should have little cost to the organism. Initial indirect support for this proposition comes from developmental work, showing that the looming bias emerges early on - it has been observed among infants as young as 4 months of age (Freiberg, Tually, \& Crassini, 2001). This suggests the looming bias may not require learning, higher-order cognitive processing, or ample cognitive resources.

Conversely, Wann, Poulter, and Purcell (2011) found that children could not reliably detect visually approaching stimuli past a specific speed threshold, as compared to adults who were able to detect the visual stimuli in all speed conditions. Given that children's neural mechanisms for detecting visually looming objects are not fully developed, it is unclear if the results are due to a lack of resources or to not having a fully mature neural system. To directly test whether the looming bias is effortless or effortful among adults, one would have to limit available mental resources and assess the outcomes of this manipulation on the looming bias among adult participants.

Limiting mental resources has already been found to negatively affect processing of environmental information by making it more challenging to focus attention, thereby delaying response times (Furley, Bertrams, Englert, \& Delphia, 2013; Lee, Lee, \& Boyle, 2007). For example, drivers who were under a cognitive load took longer to identify potential dangers in their way, braked later, and ended up being closer to the approaching danger (shorter time-tocollision; Jamson \& Merat, 2005). There is also evidence of load-induced blindness, where increasing a cognitive load led to an inability to detect certain stimuli in the environment (Macdonald \& Lavie, 2008). Even cognitive load in a different modality (e.g., visual perceptional load) can lead to a failure to detect and process auditory stimuli (Raveh \& Lavie, 2015). These findings suggest that increasing cognitive load could lead to a failure to detect looming dangers in the environment.

Alternatively, research also suggests that cognitive load could actually strengthen a bias. For example, Holmes, Mogg, de Fockert, Nielsen, and Bradley (2014) had participants memorize random strings of numbers to increase cognitive load as they were completing a version of the dot-probe task: participants had to indicate whether an arrow was pointing up or down following threatening or neutral faces. Participants exhibited a greater attentional bias for threatening stimuli under a high cognitive load, as compared to lower cognitive load, suggesting that cognitive load could actually result in a stronger rather than weaker looming bias, consistent with the margin-of-safety theory.

The current study tests both the margin-of-safety theory with regard to the looming bias (low mental resources will 
increase the looming), and whether looming is effortful or effortless. Based on the margin-of-safety theory, we predicted that (1) participants under a high cognitive load (low availability of cognitive resources) would exhibit a stronger looming bias when responding to auditory signals indicating an approaching object. If the looming bias evolved as a means to better prepare for approaching danger, then we should only observe differences in responses to approaching sounds and not receding or stationary sounds. Based on Neuhoff et al. (2012) we further predicted that (2) the looming bias is an effortless process and will occur even when cognitive resources are low.

\section{Method}

\section{Participants}

Fifty-seven undergraduate students at a large Midwestern university ( 35 males; $M_{\text {age }}=19.44, S D=1.6$ ) participated in the study for course credit. No participants reported problems with their hearing. Participants were not informed of the true intentions of the study until after the experiment was completed.

\section{Stimulus and apparatus}

The looming task was run using PsyScope (Cohen, MacWhinney, Flatt, \& Provost, 1993) on a Macintosh computer. The program recorded reaction times for the participant's responses to the three different types of sounds used in the study (looming, receding, and stationary). Participants were instructed to press a button on a response box when they perceived a sound that corresponded to an object about to make contact with them. A trial proceeded as follows: The computer displayed "READY" on the screen for $1 \mathrm{~s}$ to indicate to the participant the start of a trial, one of the three sounds (approaching, receding, and stationary) was randomly played for $2 \mathrm{~s}$. Once the participant responded to the sound by hitting a button, the "READY" screen appeared again for $1 \mathrm{~s}$ before the next trial began.

The sound used to simulate movement was a sine-wave tone at $440 \mathrm{~Hz}$ presented diotically for a duration of $2 \mathrm{~s}$ via headphones. To simulate movement, the amplitude of the sound either decreased from approximately $80 \mathrm{~dB}$ SPL (sound pressure level) to $65 \mathrm{~dB}$ SPL using a linear fade out, simulating an object "receding" from the listener (there were ten such trials), or increased from approximately $65 \mathrm{~dB}$ SPL to $80 \mathrm{~dB}$ SPL using a linear fade in, simulating an object "approaching" the listener (there were ten such trials). An additional five trials were presented in which the amplitude was constant (at approximately $80 \mathrm{~dB}$ SPL) to simulate a stationary object. Stationary and receding sounds were used to examine whether cognitive load affected only looming sounds or all sounds. In total there were 25 task trials in each block.

Previous research on the auditory looming bias has used two types of sound stimuli. In one, researchers used 3-D sound sources to assess participants' ability to evaluate object's time of arrival (e.g., Neuhoff et al., 2012; Riskind et al., 2014). In the other, researchers used increasing and decreasing sound amplitude to simulate movement and assess participants' ability to evaluate amplitude change (e.g., Tajadura-Jiménez, Väljamäe, Asutay, \& Västfjäll, 2010). Our current stimuli combines these two methods by using increasing or decreasing amplitude of the sound (for a similar approach, see Neuhoff \& Wess, 1998) and asking participants to indicate when they think an object is about to make contact with them. Previous research suggests that amplitude change is the most reliable cue for auditorily simulating object motion (Rosenblum, Carello, \& Pastore, 1987). Furthermore, Seifritz et al. (2002) found that such stimuli activate regions in the brain involved in auditory motion perception. Therefore, even though the acoustic stimulus did not physically change location, participants likely experienced the illusion of motion aurally.

Before the first (out of two) blocks of the looming task, participants were randomly assigned to one of two load conditions: low load, where they were asked to memorize two digits, or a high load condition, where they were asked to memorize seven digits. The numbers were presented at the center of the computer screen for $20 \mathrm{~s}$. This numbermemorization task has been shown in previous studies to decrease cognitive resources in the high load condition (Mann \& Ward, 2004). Participants were told that they needed to memorize the numbers and would be asked to recall the numbers at the end of the block. After $20 \mathrm{~s}$, the numbers disappeared, the "READY" screen appeared for $1 \mathrm{~s}$, and then the sound stimulus automatically played over the headphones for $2 \mathrm{~s}$. Once the participant responded, the "READY" screen appeared. When the block was finished, the participants were asked to type in the numbers they memorized.

Before proceeding to the next block of the looming task (and the other load condition), participants completed a distractor task consisting of a battery of online questionnaires for $10 \mathrm{~min}$. Previous studies have shown $10 \mathrm{~min}$ to be an adequate amount of time for participants to regenerate cognitive resources that have been depleted by a cognitively demanding task, even when participants were completing questionnaires (Tyler \& Burns, 2008). If participants finished before the 10-min period was over, the researcher instructed them to wait at their computer for the remainder of the time. The 10-min was used to decrease the chance of any carryover effects, which have been found in previous studies where participants expend mental resources (e.g., Wan \& Agrawal, 2011). At the end of $10 \mathrm{~min}$ participants were asked to memorize the digits in the other load condition and then complete another block of the looming task. 


\section{Design and procedure}

A within-subjects design was used in which participants completed the looming task once under high and once under low cognitive load condition (completion order was counterbalanced across participants). After signing the consent form, participants completed two practice blocks, which consisted of ten sound trials each (four approaching, four receding, and two stationary sounds), to familiarize themselves with the task. Participants were then allowed to ask questions if they needed further clarification of the task. No participants reported issues with understanding the looming task. After the practice blocks, participants completed one block of the actual looming task under either a low or a high cognitive load. Following the first block, participants completed the distractor task (i.e., completing questionnaires for $10 \mathrm{~min}$ ), and then completed another block of the looming task under the other cognitive load condition. Participants were then debriefed and awarded credit. The study took an average length of $20 \mathrm{~min}$ to complete.

\section{Results}

Participants' response times (RTs) in milliseconds (ms) for the 25 sound trials in each looming block were analyzed. Six scores were calculated per participant: mean RTs to the approaching sounds (i.e., the illusion of motion elicited by changes in increased amplitude), mean RTs to the receding sounds, and mean RTs to the stationary sounds in both the high and the low cognitive load conditions. Smaller numbers for the RTs of the looming sounds indicate that the participant judged the object was about to make contact with them when it was actually further away (i.e., "far"). Larger values of the approaching sound RTs indicate that the participant judged the object to be close or "near" to making contact with them when they were actually closer. We recognize the lack of precision in our use of terms like "near" and "far" to refer to the distance of the sound experienced by the participants when there was, in fact, no physical motion of the acoustic signal; we use these terms simply to facilitate comprehension in the reader.

Participants were excluded from data analysis if their reaction times were over 2,000 ms or they failed to follow instructions during the study (such as beginning the second looming block before $10 \mathrm{~min}$ had elapsed). In total, 11 participants were excluded from analysis, resulting in 46 participants included in the analysis reported below.

To test our main hypothesis, we ran a 3 (Sound Type [looming, receding, stationary]) $\times 2$ (Cognitive Load [high, low]) analysis of variance (ANOVA). The ANOVA revealed a main effect for sound type $F(2,44)=133.62, p<.001$, $\mathrm{p}^{2}=.86$, as well as an interaction between sound type and cognitive load $F(2,44)=5.06, p=.011, \mathrm{p}^{2}=.19$. There was no main effect for cognitive load. The significant effect for sound type can be attributed to the nature of the looming task. Because participants had to wait for the looming sounds to approach them before responding, these RTs were significantly longer than those of the other two sound types. Thus, grand mean RTs cannot be compared accurately across the different sound types. The stationary and receding sounds were included in the study to examine whether the effect of cognitive load on sound perception was unique to looming sounds.

Indeed, pairwise comparisons revealed that the difference between high and low load was significant only in the looming condition $(p=.04)$, such that participants' RTs under a high cognitive load (i.e., fewer cognitive resources) were lower $(M=1476.09, S D=297.63)$ than the mean RTs for the looming sounds under a low cognitive load $(M=$ $1535.79, S D=276.13$ ). There was no significant difference in the two other conditions (see Fig. 1a and b). In other words, participants with fewer cognitive resources judged an approaching sound to be making contact with them sooner (when it was actually "farther" away) compared to participants with more cognitive resources. These results suggest that there was not a general response bias affecting participants' judgments of distance from auditory cues, but rather a bias was observed only under conditions of looming sounds, as had been previously observed by Neuhoff (2001).

We also examined the possible effects of positive and negative affect and order of completion (high load first vs. low load first) on the looming bias. Positive and negative affect were assessed using the PANAS (Watson, Clark, \& Tellegen, 1988), which was a part of the battery of questions administered between the two looming blocks. There were no significant main effects or interactions for positive and negative affect or order (all $F$ 's $\leq 3.00$, and all $p$ 's $>.05$ ).

\section{Discussion}

The looming bias is the tendency to perceive approaching objects as closer than they actually are (Neuhoff, 2001). According to the margin-of-safety theory, the bias is thought to provide an organism with additional time to prepare for a potential threat (e.g., Bach et al., 2008; Neuhoff et al., 2012). Although previous research has examined how certain physical factors influence the looming bias (e.g., sex, physical fitness, age, and species; Neuhoff et al., 2012; Wann et al., 2011), almost no research to date has examined the influence of psychological factors on the looming bias as we have in the present study. Specifically we were interested in whether the availability of cognitive resources affects the strength of the looming bias and whether or not the looming bias is resource dependent. To do that we manipulated the availability of 
a

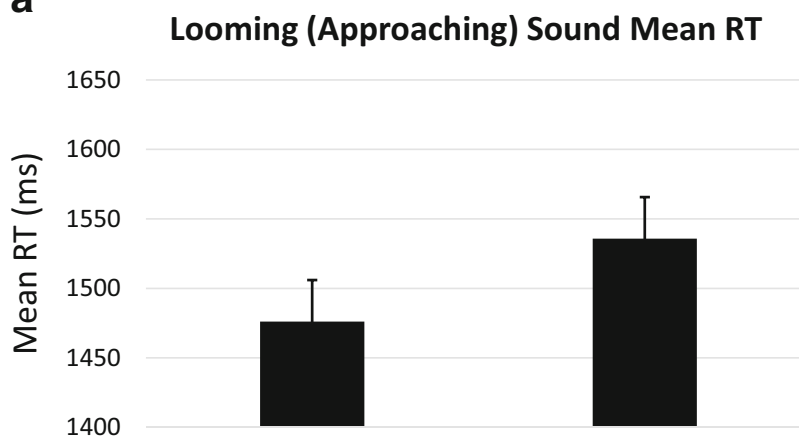

High Cognitive Load Low Cognitive Load

b

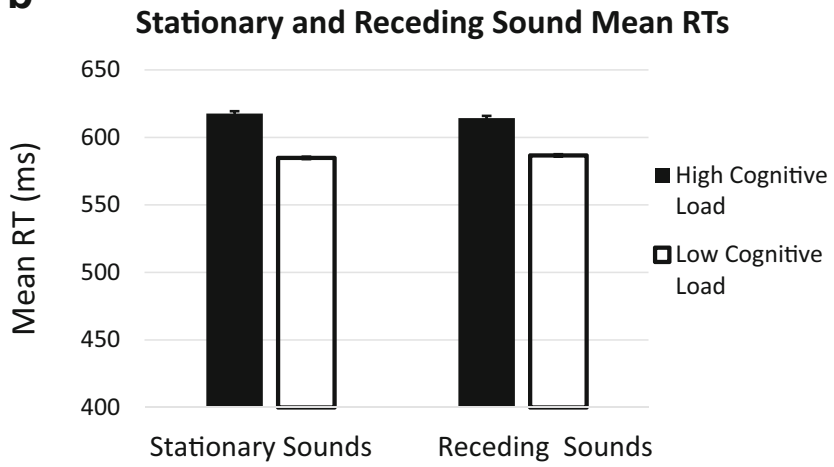

Fig. 1 a. Difference in mean response time (RT) for participants in a high and low cognitive load for looming (approaching) sounds. Smaller RTs reflect a stronger bias. Participants had a significantly stronger looming bias while under a high cognitive load as compared to those with a low cognitive load. b Difference in mean response time (RT) for participants in a high and low cognitive load for stationary and receding sounds. There were no significant differences in RTs for either of the stationary or the receding sounds between high and low cognitive loads

cognitive resources with a digit span memorization task while participants completed two blocks of the looming task. Results showed that people under high cognitive load-when fewer cognitive resources are available - had a significantly stronger looming bias (as determined by shorter RTs) as compared to people under low cognitive load. This effect was not observed with stationary or receding object sounds.

Our findings are consistent with the margin-of-safety theory (Neuhoff et al., 2012). When participants in our study had fewer cognitive resources, and hence were more vulnerable, or not as capable of handling a potential danger or threat, they exhibited a stronger looming bias. That is, they tended to judge an object to be closer than it actually was. An alternative explanation for our findings could be that the cognitive load affected all distance estimates. However, the lack of significant differences for the perceptions of non-looming sounds under a high versus low cognitive load, makes this idea less likely.

Our results also suggest that the looming bias is effortless, as opposed to effortful. Neuhoff et al. (2012) suggested that the looming bias would have little cost to the individual. Being dependent on cognitive resources would make the looming bias less adaptive, because people under threat or danger would need to allocate their resources to other tasks (like running or fighting; Edmunds, 1974). By not requiring many resources, the looming bias potentially allows people to detect threats, while also allocating the needed resources to dealing with the threat. Even when participants in the present experiment had fewer cognitive resources they exhibited a looming bias, supporting the hypothesis of Neuhoff et al. (2012).

Our findings also support the behavioral urgency hypothesis (Franconeri \& Simsons, 2003), which suggests that special attention will be given to stimuli in the environment that might require the organism to run away or defend itself. Looming objects are likely to pose a bigger threat and require immediate action, as opposed to receding or stationary objects. This would explain why the bias was only observed in the current study for looming objects and not for stationary or receding objects, which is consistent with previous studies on the looming bias (Neuhoff, 2001). Individuals do not need more time to prepare for an object moving away from them because it is not immediately threatening. Even though the stimulus (a sine wave tone) used in our study may not be considered behaviorally urgent, when the tone begins to increase in amplitude it becomes behaviorally urgent because it is perceived as an object approaching the individual. The fact that we found the bias even when using a relatively simple and impoverished stimulus in the laboratory suggests that it is highly likely that similar results will be found in more realistic settings with a naturally occurring threatening noise. Future studies will have to examine this possibility.

Our results help shed light on previous research that investigated the underlying mechanism of the looming bias. Skarratt, Gellatly, Cole, Pilling, and Hulleman (2014) have provided evidence that looming and receding motion attract attention equally. They concluded that because looming and receding motion grasp attention in a similar way, the looming bias is dependent on enhanced processing rather than on paying more attention. They suggested that this enhanced processing takes place in the motor system (Skarratt et al., 2014). Our results suggest that this enhanced processing is probably not based on conscious, complex, cognitive processes that are dependent on mental resources, but rather on a more automatic process, as the looming bias took place even when cognitive resources were reduced.

A potential limitation of our study is that the use of distance judgments based on amplitude change does not allow us to compare RTs for all three sound stimuli as can be done with distance judgments of 3-D sound sources (e.g., Neuhoff et al., 2012). However, what was important in the current study was not the ability to compare RTs across the three sound types, but rather the ability to compare RTs in the different cognitive load conditions for each sound type individually.

Certain emotional states, such as anxiety and fear, can affect the looming bias (Gagnon et al., 2013; Riskind et al., 
2014). Additionally, certain types of mood have been shown to influence auditory processing (e.g., negative mood affecting perceived sound loudness; Siegel \& Stefanucci, 2011). Engaging in the cognitive load task in the present experiment may have induced certain emotions in participants, and these emotions, rather than the load, may have resulted in the observed effects. To rule out this possibility we ran a statistical analysis controlling for PANAS (Watson et al., 1988) scores, and the results showed no main effects or interaction for emotional state. This finding supports our interpretation that cognitive load rather than affect influenced performance in the current study.

Despite these limitations the current study allowed us to demonstrate that limited availability of cognitive resources affects the perception of looming objects. Increasing participants' cognitive load decreased response times for looming objects, or, in other words, the greater the cognitive load a participant experienced, the stronger his or her looming bias was. Our findings provide support for both the margin-ofsafety theory and the behavioral urgency hypothesis, adding psychological vulnerability to the factors affecting the looming bias.

Acknowledgments This research was funded by an Undergraduate Research Award from the University of Kansas Center for Undergraduate Research.

\section{References}

Abrams, R. A., \& Christ, S. E. (2006). Motion onset captures attention: A rejoinder to Franconeri and Simons (2005). Perception \& Psychophysics, 68, 114-117.

Bach, D.R., Schächinger, H., Neuhoff, J.G., Esposito, F., Di Salle, F., Lehmann, C.,... Seifritz, E. (2008). Rising sound intensity: An intrinsic warning cue activating the amygdala. Cerebral Cortex, 18, 145-150. doi:10.1093/cercor/bhm040

Barkow, J. H., Cosmides, L., \& Tooby, J. (Eds.). (1995). The adapted mind: Evolutionary psychology and the generation of culture. New York: Oxford University Press.

Cacioppo, J., \& Fredberg, L. (2012). The perceiving mind: Sensation and perception. In Crabtree (Ed.), Discovering Psychology: The science of mind, briefer version (1st ed., pp. 109-163). Belmont: Wadsworth.

Cohen, J., MacWhinney, B., Flatt, M., \& Provost, J. (1993). PsyScope: An interactive graphic system for designing and controlling experiments in the psychology laboratory using Macintosh computers. Behavior Research Methods, Instruments, \& Computers, 25, 257271. doi:10.3758/BF03204507

Cole, G. G., \& Kuhn, G. (2010). Attentional capture by object appearance and disappearance. The Quarterly Journal of Experimental Psychology, 63, 147-159. doi:10.1080/17470210902853522

Edmunds, M. (1974). Defense in Animals: A Survey of Anti-predator Defenses. Harlow: Longman.

Franconeri, S. L., \& Simons, D. J. (2003). Moving and looming stimuli capture attention. Perception \& Psychophysics, 65, 999-1010.

Freiberg, K., Tually, K., \& Crassini, B. (2001). Use of an auditory looming task to test infants' sensitivity to sound pressure level as an auditory distance cue. British Journal of Developmental Psychology, 19, 1-10. doi:10.1348/026151001165903

Furley, P., Bertrams, A., Englert, C., \& Delphia, A. (2013). Ego depletion, attentional control, and decision making in sport. Psychology of Sport and Exercise, 14, 900-904. doi:10.1016/j.psychsport.2013.08.006

Gagnon, K. T., Geuss, M. N., \& Stefanucci, J. K. (2013). Fear influences perceived reaching to targets in audition, but not vision. Evolution and Human Behavior, 34, 49-54. doi:10.1016/j.evolhumbehav. 2012.09.002

Ghazanfar, A. A., Neuhoff, J. G., \& Logothetis, N. K. (2002). Auditory looming perception in rhesus monkeys. Proceedings of the National Academy of Sciences of the United States of America, 99, 1575515757. doi:10.1073/pnas.242469699

Holmes, A., Mogg, K., de Fockert, J., Nielsen, M. K., \& Bradley, B. P. (2014). Electrophysiological evidence for greater attention to threat when cognitive control resources are depleted. Cognitive, Affective, \& Behavioral Neuroscience, 14, 827-835. doi:10.3758/s13415013-0212-4

Jamson, A. H., \& Merat, N. (2005). Surrogate in-vehicle information systems and driver behaviour: Effects of visual and cognitive load in simulated rural driving. Transportation Research Part F: Traffic Psychology and Behaviour, 8, 79-96. doi:10.1016/j.trf.2005.04.002

LeDoux, J. E. (1994). The amygdala: Contributions to fear and stress. Seminars in Neuroscience, 6, 231-237.

Lee, Y. C., Lee, J. D., \& Boyle, L. N. (2007). Visual attention in driving: The effects of cognitive load and visual disruption. Human Factors: The Journal of the Human Factors and Ergonomics Society, 49, 721-733. doi:10.1518/001872007X215791

Macdonald, J. S. P., \& Lavie, N. (2008). Load induced blindness. Journal of Experimental Psychology: Human Perception and Performance, 34, 1078-1091. doi:10.1037/0096-1523.34.5.1078

Mann, T., \& Ward, A. (2004). To eat or not to eat: Implications of the attentional myopia model for restrained eaters. Journal of Abnormal Psychology, 113, 90-98. doi:10.1037/0021-843X.113.1.90

Neuhoff, J. G. (2001). An adaptive bias in the perception of looming auditory motion. Ecological Psychology, 13, 87-110. doi:10.1207/ S15326969ECO1302_2

Neuhoff, J. G., Long, K. L., \& Worthington, R. C. (2012). Strength and physical fitness predict the perception of looming sounds. Evolution and Human Behavior, 33, 318-322. doi:10.1016/j.evolhumbehav. 2011.11.001

Neuhoff, J. G., Planisek, R., \& Seifritz, E. (2009). Adaptive sex differences in auditory motion perception: Looming sounds are special. Journal of Experimental Psychology: Human Perception and Performance, 35, 225-234. doi:10.1037/a0013159

Neuhoff, J. G., \& Wess, J. (1998). Preferential detection of rising versus falling intensity. The Journal of the Acoustical Society of America, 103, 2848.

Raveh, D., \& Lavie, N. (2015). Load-induced inattentional deafness. Attention, Perception, \& Psychophysics, 77, 483-492. doi:10. 3758/s13414-014-0776-2

Riskind, J. H., Kleiman, E. M., Seifritz, E., \& Neuhoff, J. (2014). Influence of anxiety, depression and looming cognitive style on auditory looming perception. Journal of Anxiety Disorders, 28, 45-50. doi:10.1016/j.janxdis.2013.11.005

Rosenblum, L. D., Carello, C., \& Pastore, R. E. (1987). Relative effectiveness of three stimulus variables for locating a moving sound source. Perception, 16, 175-186. doi:10.1068/p160175

Seifritz, E., Neuhoff, J.G., Bilecen, D., Scheffler, K., Mustovic, H., Schächinger, H., ... \& Di Salle, F. (2002). Neural processing of auditory looming in the human brain. Current Biology, 12, $2147-$ 2151. doi:10.1016/S0960-9822(02)01356-8

Siegel, E. H., \& Stefanucci, J. K. (2011). A little bit louder now: Negative affect increases perceived loudness. Emotion, 11, 1006-1011. doi: $10.1037 / \mathrm{a} 0024590$ 
Skarratt, P. A., Gellatly, A. R. H., Cole, G. G., Pilling, M., \& Hulleman, J. (2014). Looming motion primes the visuomotor system. Journal of Experimental Psychology: Human Perception and Performance, 40, 566-579. doi:10.1037/a0034456

Sorg, B. A., \& Whitney, P. (1992). The effect of trait anxiety and situational stress on working memory capacity. Journal of Research in Personality, 26, 235-241.

Tajadura-Jiménez, A., Väljamäe, A., Asutay, E., \& Västfjäll, D. (2010). Embodied auditory perception: The emotional impact of approaching and receding sound sources. Emotion, 10, 216-229. doi: 10.1037/a0018422

Tyler, J. M., \& Burns, K. C. (2008). After depletion: The replenishment of the self's regulatory resources. Self and Identity, 7, 305-321. doi:10. 1080/15298860701799997

von Mühlenen, A., \& Lleras, A. (2007). No-onset looming motion guides spatial attention. Journal of Experimental Psychology: Human
Perception and Performance, 33, 1297-1310. doi:10.1037/00961523.33.6.1297

Wan, E. W., \& Agrawal, N. (2011). Carryover effects of self-control on decision making: A construal-level perspective. Journal of Consumer Research, 38, 199-214. doi:10.1086/658471

Wann, J. P., Poulter, D. R., \& Purcell, C. (2011). Reduced sensitivity to visual looming inflates the risk posed by speeding vehicles when children try to cross the road. Psychological Science, 22, 429-434. doi: $10.1177 / 0956797611400917$

Waterhouse, J., \& Campbell, I. (2011). Reflexes: Principles and properties. Anaethesia \& Intensive Care Medicine, 12, 214219.

Watson, D., Clark, L. A., \& Tellegen, A. (1988). Development and validation of brief measures of positive and negative affect: The PANAS scales. Journal of Personality and Social Psychology, 54, 1063-1070. doi:10.1037/0022-3514.54.6.1063 